\title{
Comments on 'Sentinel Lymph Node Mapping with Isosulfan Blue or Indocyanine Green in Colon Cancer Shows Comparable Results and Identifies Patients with Decreased Survival: A Prospective Single-Center Trial'
}

\author{
Gabriel Liberale ${ }^{1}$
}

Published online: 29 December 2017

(C) Société Internationale de Chirurgie 2017

First, I want to congratulate the authors for their excellent work comparing two techniques for the detection of sentinel lymph nodes (SLN) in colon cancer [1]. Weixler et al. compare the standard 'blue dye' technique using Isosulfan blue with a 'fluorescence imaging technique using a nonspecific fluorophore.' However, we are concerned that the authors fail to distinguish the differences between 'indocyanine green fluorescence imaging' (ICG-FI) and fluorescence imaging (FI) with the IRDye $800 \mathrm{CW}$ fluorophore used in their study. There is no correlation between these two molecules apart from the fact that both are used as 'fluorescent probes' in the near infrared (NIR) spectrum for FI. In fact, as mentioned in the paper by the authors, IRDye $800 \mathrm{CW}$ is not FDA approved for 'in vivo' human use. Conversely, ICG has been FDA approved since the early 1960s and is recognized as a safe NIR probe for clinical use in several indications. The main indication is being its use as contrast agent after intravenous injection [2,3]. By using the same term for these two different dyes, the results presented in this study may be confused with those of our published study comparing the standard ex vivo blue dye technique using patent blue with ICG-FI (not cited in Weixler et al. 2017) [4]. Finally, the authors also argue that HSA800, a combination of human serum albumin and IRDye $800 \mathrm{CW}$, contains indocyanine green at a ratio of
1:3 (ICG:Albumin). This is incorrect as HSA800 contains only IRDye $800 \mathrm{CW}$ at a ratio of 1:3 (IRDye 800CW:Albumin).

Therefore, in an effort to avoid confusion for the scientific community, we believe it is important to point out these important inconsistencies in terminology.

\section{References}

1. Weixler B, Rickenbacher A, Raptis DA et al (2017) Sentinel lymph node mapping with isosulfan blue or indocyanine green in colon cancer shows comparable results and identifies patients with decreased survival: a prospective single-center trial. World J Surg 41:2378-2386. https://doi.org/10.1007/s00268-017-4051-2

2. Liberale G, Bourgeois P, Larsimont D et al (2017) Indocyanine green fluorescence-guided surgery after IV injection in metastatic colorectal cancer: a systematic review. Eur J Surg Oncol 43:1656-1667

3. Koh FH, Tan KK (2016) Fluorescent angiography used to evaluate the perfusion status of anastomosis in laparoscopic anterior resection. Ann Surg Oncol 23(Suppl 5):692

4. Liberale G, Vankerckhove S, Galdon MG et al (2016) Sentinel lymph node detection by blue dye versus indocyanine green fluorescence imaging in colon cancer. Anticancer Res $36: 4853-4858$
Gabriel Liberale

Gabriel.liberale@bordet.be

1 Department of Digestive Surgical Oncology, Jules Bordet Institute, Université Libre de Bruxelles (U.L.B.), Brussels, Belgium 